\title{
Theory of Acoustic Excitations in Colloidal Suspensions
}

\author{
Xiaodun Jing, Ping Sheng, and Minyao Zhou \\ Exxon Research \& Engineering Co., Route 22 East, Annandale, New Jersey 08801
}

(Received 19 September 1990)

\begin{abstract}
Recent Brillouin scattering experiments have revealed the surprising existence of two acoustic modes in colloidal suspensions of solid spheres. Through an analytic Green's-function calculation and numerical simulations, we show that the higher-frequency mode is associated with the solid-sphere antiresonances, and the lower-frequency mode arises from the coupling of internal resonances between neighboring spheres. Our theory yields excellent agreement with the measured dispersion relations and offers and explanation for the observed frequency gaps in the excitation spectra.
\end{abstract}

PACS numbers: $82.70 . \mathrm{Dd}, 03.40 . \mathrm{Kf}, 62.65 .+\mathrm{k}, 68.45 . \mathrm{Nj}$

It is the conventional wisdom that an isotopic, homogeneous elastic solid has one longitudinal and two shear modes, and a fluid has only one longitudinal acoustic mode. For inhomogeneous materials, however, Biot ${ }^{1}$ has predicted that it is possible to have two longitudinal modes in a fluid-saturated porous medium where the faster wave travels predominantly in the solid frame and the slow wave travels predominantly in the liquid. These predictions were indeed confirmed experimentally. ${ }^{2}$ Here the key element is that both the solid and the fluid phases must form continuous networks so as to provide paths of propagation for the two modes. Hence in colloidal suspensions, where only the fluid phase is continuous, it is the general expectation that only one longitudinal-acoustic mode exists. At low frequencies, such expectation has been supported by both theoretical calculations ${ }^{3,4}$ and experimental observation. ${ }^{3}$

Recent Brillouin scattering experiments ${ }^{5}$ on colloidal suspensions, consisting of monodisperse polymethylmethacrylate (PMMA) spheres (370 or $680 \mathrm{~nm}$ in diameter) dispersed in oil, have yielded the surprising results that show whereas in the low-frequency regime there is indeed only one acoustic mode, at angular frequencies higher than $\pi c_{l} / d$, where $c_{l}$ is the fluid wave speed and $d$ the sphere diameter, there exist two distinct longitudinal modes whose characteristics vary with the concentration of the solid spheres. These experimental results directly challenge our understanding of one of the most basic topics in classical physics. In addition, since the occurrence of the two modes coincides with the regime of strong resonant scattering, even the existence of propagating modes is a surprise and contrary to the conventional view that only diffusive transport $^{6}$ exists in the strong-scattering regime.

In this Letter, we use a Green's-function calculation and numerical simulations to clarify the physical origin of the two acoustic modes in colloidal suspensions. It is shown that the two acoustic excitations are quasimodes with finite lifetimes. Whereas the high-frequency mode is associated with the antiresonances of a single sphere in liquid, the low-frequency mode arises from coupling of internal resonances between neighboring spheres. The latter is interpretable as a coupled interfacial Stone- ley wave ${ }^{7}$ which propagates between adjacent spheres through the decaying portion of the excitation in the fluid. Excellent agreement is obtained between theory and experiment.

A colloidal suspension is characterized by the dispersion microgeometry where each spherical solid particle is individually enveloped by the fluid. This microgeometry is to be contrasted with the alternate possibilities in which the particles can cluster and touch. To carry out Green's-function calculations, we take into account this strong short-range correlation between the solid and fluid phases by considering as the basic scattering unit a coated sphere, where the fluid coating thickness $s$ is determined by the solid particle concentration $\phi=(1+2 s /$ $d^{-3}$. To calculate the effective macroscopic properties of tive system, the coated sphere is embedded in a homogenized effective medium composed of similar units of coated spheres, with the effective-medium speed(s) $\bar{c}$ of the medium to be determined by some self-consistent condition. In the coherent-potential approximation ${ }^{8}$ (CPA), such a condition is achieved by requiring the vanishing of the forward-scattering amplitude $f(0)$ through the adjustment ${ }^{9}$ of $\bar{c}$. The condition is noted to be self-consistent in the following sense. If we let $G$ denote the exact Green's function for an acoustic wave in the colloidal system, then ${ }^{8}$

$$
G=G_{0}+G_{0} T G_{0}
$$

where $G_{0}$ is the Green's function for the homogeneous effective medium and $T$ denotes the exact total scattering operator (including all the multiple scattering between particles). By expressing $G_{0}=\left(p^{2}-q^{2}\right)^{-1}$, with $q=\omega / \bar{c}$ and $p$ the momentum variable, the CPA condition for Eq. (1) is $\langle T\rangle=0$ through the adjustment of $q$. When that happens, $\langle G\rangle=G_{0}$, and $q$ is identified as the wave vector of the excitation. Since $\langle T\rangle \simeq n t$ in the weak-scattering limit, where $t$ is the single-coated-sphere scattering operator and $n$ the sphere density, $f(0)=t_{q q}$ $=0$ is therefore the condition not only for determining $q$, but also for justifying the weak-scattering assumption so that the problem may be cast in the single-scatterer form in the first place.

In this work, a generalized version of the CPA condi- 
tion is used to identify the excitation modes in colloidal suspensions. That is, instead of requiring $\langle T\rangle=0$, we wish to identify the $q$ value(s) at which the singlecoated-sphere scattering is at a local minimum for a wave of given frequency $\omega$. The fact that the scattering does not vanish means $\langle G\rangle=\left(p^{2}-q^{2}-\Sigma\right)^{-1}$, where the self-energy $\Sigma \simeq n t$ to the first order in scattering strength. Here the real part of $\Sigma$ renormalizes $q^{2}$, and the imaginary part of $\Sigma$ gives the mean free path, or lifetime, of the quasimode(s).

To calculate the Green's function for a single coated sphere embedded in an effective medium, we start from the elastic wave equation ${ }^{7}$ for each of the three homogeneous regions:

$$
\frac{\partial^{2} \mathbf{u}}{\partial t^{2}}=c_{t}^{2} \nabla^{2} \mathbf{u}+\left(c_{p}^{2}-c_{t}^{2}\right) \nabla(\nabla \cdot \mathbf{u}),
$$

where $\mathbf{u}$ is the displacement, $c_{p}$ is the longitudinal and $c_{t}$ the shear wave velocities. For the fluid coating and the effective medium, $c_{t}=0$. By decomposing $\mathbf{u}=-\nabla \psi$ $+\nabla \times \mathbf{A}$, with the vector potential $\mathbf{A}$ being further expressed as $\mathbf{A}=\boldsymbol{\nabla} \times \mathbf{r} \xi$, we get a pair of Helmholtz equations for $\psi$ and $\xi$. Here $\psi$ is directly proportional to the density variation, $\boldsymbol{\nabla} \cdot \mathbf{u}$. Since in Brillouin scattering the light is coupled to thermal excitations through refractive-index changes induced by density variations, the relevant Green's function is that for measuring the $\psi$ response to a point scalar source, i.e.,

$$
\left[\nabla^{2}+\omega^{2} / c_{p}^{2}(\mathbf{r})\right] g_{\psi}\left(\mathbf{r}, \mathbf{r}^{\prime}\right)=\delta\left(\mathbf{r}-\mathbf{r}^{\prime}\right),
$$

where $c_{p}(\mathbf{r})=c_{s}=2.7 \times 10^{5} \mathrm{~cm} / \mathrm{sec}$ for $r<d / 2, c_{p}(\mathbf{r})=c_{l}$ $=1.2 \times 10^{5} \mathrm{~cm} / \mathrm{sec}$ for $d / 2 \leq d / 2+s$, and $c_{p}(\mathrm{r})=\omega / q$ for $r \geq s+d / 2$. For the $\xi$ field, on the other hand, we have $\left(\nabla^{2}+\omega^{2} / c_{t}^{2}\right) g_{\xi}\left(\mathbf{r}, \mathbf{r}^{\prime}\right)=0$ for $r<d / 2$, where $c_{t}=1.1 \times 10^{5}$ $\mathrm{cm} / \mathrm{sec}$, and $g_{\xi}=0$ otherwise. The $g_{\psi}$ and $g_{\xi}$ are coupled by the boundary conditions, i.e., continuity of normal displacement and normal stress, and tangential stress equals zero at $r=d / 2$; and the continuity of normal displacement and stress at $r=d / 2+s$. Here we have taken the fluid to be inviscid. The effect of viscosity is investigated by numerical simulations to be described later. The Green's function $g_{\psi}\left(\mathbf{r}, \mathbf{r}^{\prime}\right)$ is obtained by solving three boundary-value problems where the source point $\mathbf{r}^{\prime}$ is located in each of the three spatial regions (sphere, coating, and the effective medium). By Fourier transforming $g_{\psi}\left(\mathbf{r}, \mathbf{r}^{\prime}\right)$, we get $g_{\psi}\left(\mathbf{p}_{1}, \mathbf{p}_{2}, q ; \omega\right)$, where the dependence on $q$ and $\omega$ is explicitly noted. Since $g=G_{0}+G_{0} t G_{0}$, the scattering operator $t$ for a single coated sphere may thus be obtained. In accordance with the discussion above, the final result of our calculation is the density of states (DOS), $-\operatorname{Im}\langle G\rangle / \pi$, evaluated with the condition of elastic scattering so that $\left|\mathbf{p}_{1}\right|=\left|\mathbf{p}_{2}\right|=p$ $=q$. Since $\langle G\rangle=\left(p^{2}-q^{2}-\Sigma\right)^{-1} \cong\left(p^{2}-q^{2}-n t\right)^{-1}$, at $p=q$ we have $G \cong-(n t)^{-1}$ so that the maxima of DOS correspond directly to minima in scattering.

In Figs. 1 (a) and 1(b) we plot the DOS as a function of dimensionless frequency, $\omega d / c_{l}$, and $q d$ for $\phi=0.38$ and 0.51 , respectively. It should be noted that there are
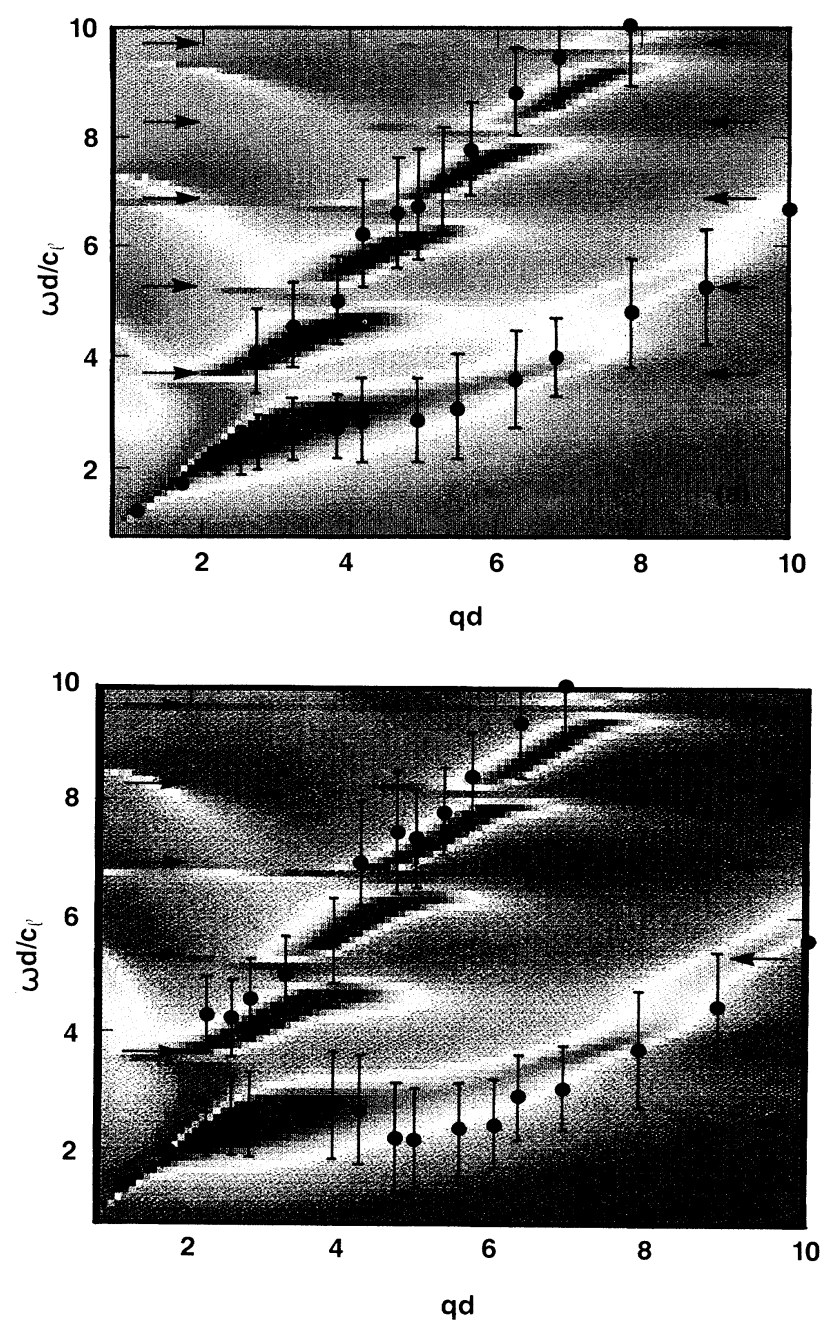

FIG. 1. Density of states (DOS) plotted as a function of $\omega d / c_{l}$ and $q d$. The magnitude of DOS is delineated by color, where high is indicated by red and low is indicated by blue. Yellow is intermediate. The dispersion relation of the fluid would be a straight line with slope 1. Experimental data are shown as solid circles. The bars indicate their frequency widths. The arrows on the sides indicate the frequency positions of the scattering cross-section peaks for a single sphere in fluid. (a) $\phi=0.38$ and (b) $\phi=0.51$.

no adjustable parameters in our calculations. Two bands of ridges are clearly seen in red and yellow. They correspond remarkably well to the experimentally observed dispersion relations for the two acoustic modes, shown as black points with bars indicating the measured frequency width. While not shown, our calculations also indicate that (1) below $\phi \simeq 0.2$ the two dispersion relations merge and become indistinguishable from the fluid dispersion relation, and $(2)$ regardless of $\phi$, at high $q(\approx 20)$ the high-frequency mode tends to converge to the fluid dispersion relation. Both are in agreement with experimental observations. The black arrows on the sides indi- 
cate the frequency positions of the peaks in scattering cross section for a single sphere in fluid. They are the key to understanding the physical origin of the two modes as shown below.

For the high-frequency mode, the positions of the arrows correspond directly with the gaps in the calculated dispersion relations. The first gap is clearly seen in the experimental results for both $\phi=0.38$ and 0.51 and the second gap and hint of a third are also visible; although for the higher gaps their frequency positions do not agree with the theory as well. What this indicates is that the high-frequency mode results from the antiresonance condition for the single-sphere scattering. The fact that the minima are nonzero implies that the excitations are quasimodes with finite lifetimes.

For the low-frequency mode, on the other hand, the frequency positions of the scattering peaks as indicated by the arrows are noted to coincide with the peaks of the DOS, and in Fig. 1(a) the calculated gap in its dispersion relation is seen to correspond reasonably well with the experimentally observed one. The low-frequency mode is therefore the direct result of single-sphere resonances, in contrast to the high-frequency mode. The minima of the scattering, as evidenced by the maxima of DOS, are the consequence of interaction between neighboring spheres and the resulting split of a single resonance mode into two, with a (nonzero) scattering minimum in between. As seen from the point of view of an individual sphere, the interaction with neighboring spheres may be represented by some particular value of the wave impedance at the coating-effective-medium interface. Since the effective-medium $q$ is treated as a variable, we are thus able to pick up the scattering minimum by scanning its value.

The relevant single-sphere resonances discussed above depend crucially on the shear modulus of the solid sphere. They are analogous to the "surface modes" of small metallic spheres ${ }^{10}$ that may be viewed as an extension of the surface plasmon to the spherical geometry. Here the relevant flat-surface excitation is the Stoneley mode for the solid-liquid interface (which disappears if the solid shear modulus vanishes). Since the highfrequency mode depends on the antiresonance condition whereas the low-frequency mode depends on the resonance condition (with interaction), they are expected to exhibit distinct behaviors as the shear modulus varies. Figure 2 demonstrates the effects of the shear modulus on the two modes. At a fixed frequency, the DOS is plotted as a function of $q$. Two peaks are clearly seen. As the shear modulus is progressively decreased, the high-frequency mode (low- $q$ peak) shows only minor perturbations, but the low-frequency mode not only shifts to higher $q$ (lower frequency for a fixed $q$ ) but also diminishes in its amplitude, indicating that the shear modulus is responsible for the strength of the lowfrequency mode. A valid physical picture that emerges is therefore one of "surface Stoneley modes" coupled

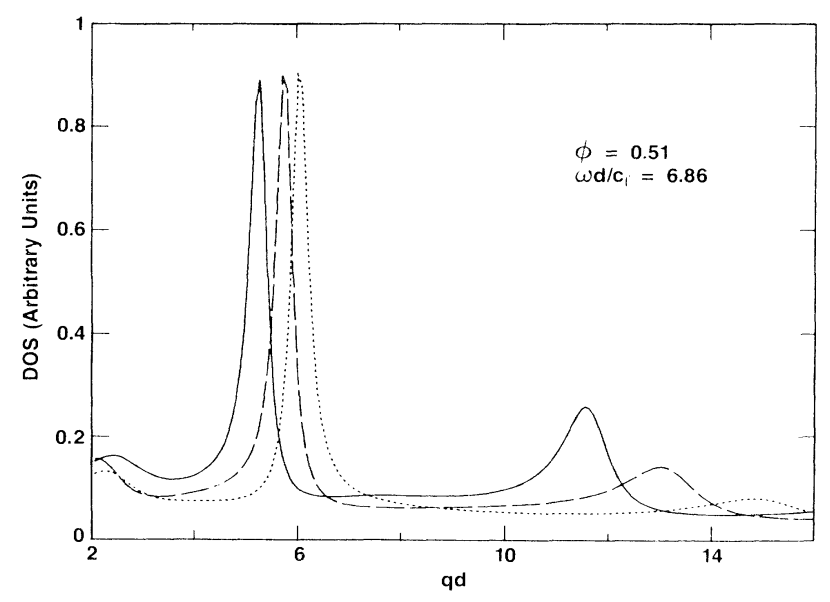

FIG. 2. DOS at a given frequency plotted as a function of $q d$. The two peaks correspond to the two quasimodes. The dashed and dotted lines correspond to calculations where the shear modulus of the solid sphere is progressively decreased from its physical value to 0.64 and 0.46 times of its physical value.

through their decaying portions in the fluid. Further support for this picture is found in the fact that both in theory and in experiment the speed of the low-frequency mode approaches that of the Stoneley wave $\left(C_{\text {Stoneley }}\right.$ $=0.86 \times 10^{5} \mathrm{~cm} / \mathrm{sec}$ ) at the large- $q$ limit, ${ }^{5}$ where the surface-curvature effect becomes negligible. In contrast, for the high-frequency mode the disappearance of shear-induced resonances only implies the disappearance of gaps in its dispersion relation.

It should be noted that the observed frequency widths always seem to be larger than those calculated, and the higher-frequency gaps do not agree well with the theoretical positions. This could be due to the effect of viscosity as well as to enhanced scattering induced by disorder, which has been homogenized in our calculation. Also, the low-frequency mode is noted to have a dip in its dispersion relation for the $\phi=0.51$ case that is not reproduced by theory. This is clearly a short-rangeorder effect, which is also absent in our present version of the calculation.

To further check our theory and the effects of fluid viscosity, we use finite differencing to simulate the wave behavior in two spatial dimensions. Here the relevant equations are the elastic wave equation for the solid region and the linearized Navier-Stokes equation for the fluid region, with the compressibility condition $\nabla \cdot \mathbf{v}$ $=-\kappa^{-1} \partial p / \partial t$, where $\mathbf{v}$ is the displacement velocity, $\kappa$ the fluid bulk modulus, and $p$ the pressure. The boundary conditions of displacement velocity continuity and traction continuity are applied across interfaces. ${ }^{11} \mathrm{~A}$ line time-harmonic source with frequency $\omega$ is placed on one side of a $600 \times 600$ grid. Solid circles are randomly placed on the grid, with twenty points across the diameter of each circle. The discretized partial differential 


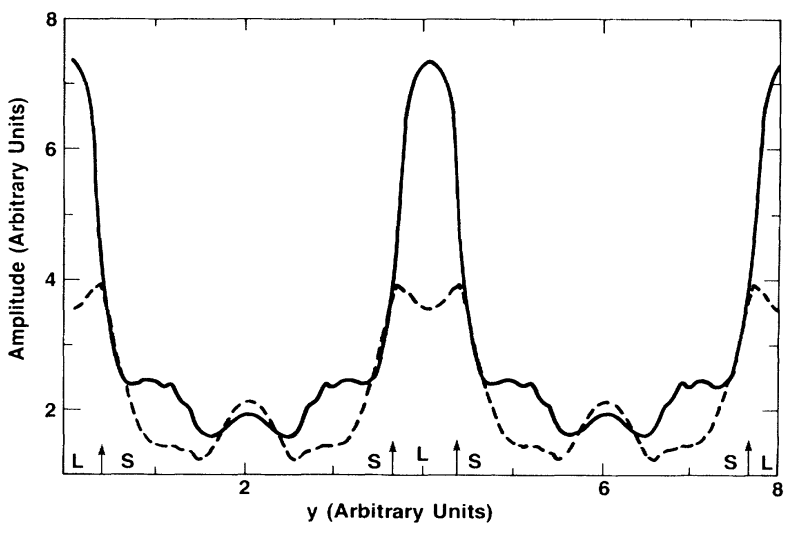

FIG. 3. Amplitude of $\boldsymbol{\nabla} \cdot \mathbf{v}$ for the two modes plotted along a line perpendicular to the direction of wave propagation. The frequency used is $\omega d / c_{l}=7.5$. The dashed line denotes the low-frequency mode. The solid line denotes the high-frequency mode. Regions of solid and liquid are indicated by $S$ and $L$, respectively, at the bottom of the figure. The low-frequency mode is noted to have a cusp at the liquid-solid interface.

equations are numerically integrated forward in time. After a suitably long integration time, the integration is stopped and the spatial distribution of $\boldsymbol{\nabla} \cdot \mathbf{v}$ is Fourier transformed into the q domain. The Fourier amplitudes can then be plotted as a function of $|q|$ for a given $\omega$. Two peaks are clearly seen, thus directly verifying the existence of the two modes in a two-dimensional system. The dispersion relations of the two modes are also qualitatively similar to that shown in Fig. 1, and the effect of decreasing the shear modulus is exactly the same as that shown in Fig. 2. We have further simulated systems with polydispersity. Similar results were obtained, indicating the general robust nature of the phenomenon.

The effect of fluid viscosity has been examined. We found that unless the viscosity is unphysically large, its presence only causes minor corrections to the positions and widths of the two peaks. ${ }^{12}$ We can thus rule out viscous coupling as a basic cause for any one of the two observed modes.

Numerical simulation enables us to obtain a microscopic picture of the two modes. Each of the two peaks in the $|q|$ plot can be separately Fourier transformed to the spatial domain. Figure 3 gives a view of the $\boldsymbol{\nabla} \cdot \mathbf{v}$ amplitude for the two modes in a line perpendicular ( $y$ direction) to the direction of wave propagation ( $x$ direction). In this case the line cuts through the center of two circles. It is seen that the amplitudes of both modes are higher in the fluid than in the solid. This is consistent with our observation that both modes result from scattering minima. Also, whereas the amplitude of the highfrequency mode crosses the solid-liquid interface smoothly, the low-frequency-mode amplitude exhibits a cusp at the interface, indicating its Stoneley-wave origin.

At present, further considerations are given to the role of short-range order and the acoustic-wave-localization characteristics. The interesting possibility that similar mode structure might be observed for electromagnetic waves propagating in metallic suspensions is also being pursued.

The authors wish to acknowledge many helpful discussions with D. A. Weitz, J. Liu, L. Ye, and Z. Q. Zhang. Thanks are due to $S$. Ferguson for help in making the color pictures.

${ }^{1}$ M. A. Biot, J. Acoust. Soc. Am. 28, 168 (1956); 28, 5848 (1956).

${ }^{2}$ T. J. Plona, Appl. Phys. Lett. 36, 259 (1980).

${ }^{3}$ L. M. Schwartz and T. J. Plona, J. Appl. Phys. 55, 3971 (1984).

${ }^{4}$ L. M. Schwartz and D. L. Johnson, Phys. Rev. B 30, 4302 (1984).

${ }^{5}$ J. Liu, L. Ye, D. A. Weitz, and P. Sheng, Phys. Rev. Lett. 65, 2602 (1990).

${ }^{6}$ See Scattering and Localization of Classical Waves in Random Media, edited by P. Sheng (World Scientific, Singapore, 1990).

${ }^{7}$ See, for example, L. M. Brekhovskikh, Waves in Layered Media (Academic, New York, 1980).

${ }^{8}$ See E. N. Economou, Green's Function in Quantum Physics (Springer-Verlag, New York, 1983), 2nd ed., p. 141.

${ }^{9}$ In the electromagnetic case, this results in the well-known Maxwell-Garnett theory for the effective dielectric constant of composites with the dispersion microgeometry. See a review by P. Sheng, in Homogenization and Effective Moduli of Materials and Media, edited by J. L. Ericksen, D. Kinderlehrer, R. Kohn, and J. L. Lions (Springer-Verlag, New York, 1986), p. 196.

${ }^{10}$ C. F. Bohren and D. R. Huffman, Absorption and Scattering of Light by Small Particles (Wiley-Interscience, New York, 1983), p. 325.

${ }^{11}$ By expressing all the solid and fluid equations as a system of coupled first-order partial differential equations, it can be shown that direct finite differencing across interfaces guarantees the boundary conditions. See B. Fornberg, Geophysics 52, 483 (1987).

${ }^{12} \mathrm{We}$ have made sure that there are at least a few points in the viscous relaxation length so that the viscous boundary layer can be resolved. 

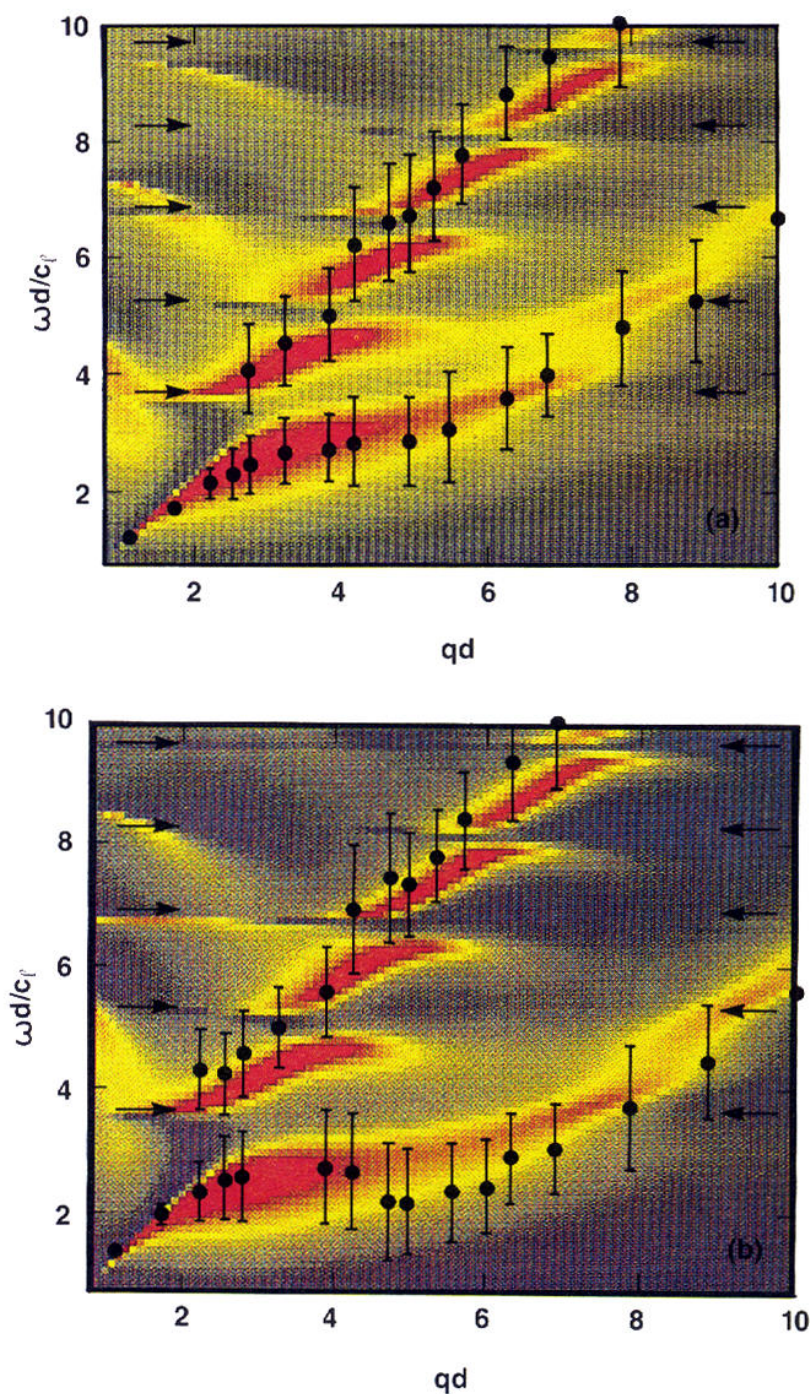

FIG. 1. Density of states (DOS) plotted as a function of $\omega d / c_{l}$ and $q d$. The magnitude of DOS is delineated by color, where high is indicated by red and low is indicated by blue. Yellow is intermediate. The dispersion relation of the fluid would be a straight line with slope 1 . Experimental data are shown as solid circles. The bars indicate their frequency widths. The arrows on the sides indicate the frequency positions of the scattering cross-section peaks for a single sphere in fluid. (a) $\phi=0.38$ and (b) $\phi=0.51$. 